\title{
Data Mining Implementation on Choosing Potential Customers Using K-Means Algorithm on PT. Koba Metal Indonesia
}

\author{
Sandi Kristianto ${ }^{1}$, Yusuf Kurnia ${ }^{2}$ \\ ${ }^{1}$ Universitas Buddhi Dharma, Teknik Informatika, Banten, Indonesia \\ ${ }^{2}$ Universitas Buddhi Dharma, Teknik Informatika, Banten, Indonesia
}

\begin{tabular}{l}
\hline SUBMISSION TRACK \\
\hline Received : February 1,2018 \\
Final Revision: February 6, 2018 \\
Available Online: February 27, 2018 \\
KEYWORD \\
\hline Potential Customers, Data Mining, Clustering, \\
K-Means, Rapidminer. \\
CORRESPONDENCE \\
\hline
\end{tabular}

Telepon: 081281500393

E-mail: sandi.kristianto@gmail.com

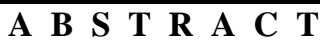

PT. Koba metal Indonesia. is one of roll-reforming cooperations who produce light-steel stuffs which is growing rapidly nowadays. One of the important thing in customer management is how a cooperation be able to preserve their customers. the effort of preserving customers becomes important for PT. Koba metal Indonesia. considering of plenty companies who commits at the same sector. To prevent the displacement of customers, knowing the potential group of customers is important, so that the company could preserve those potential customers by giving excellent service, etc. the implication of data mining could assist the company to analize the received data from sales transaction to gain potential customers data. Therefore, a designed application which could implement the data mining for choosing potential customers by clustering and algorithm K-means method is arranged. Then, the information performes with groups who is categorized into potential customers. Besides, rapminder application is also used to examine the data's accuracy of this built application design. Hereinafter, this application design is expected to assist companies to choose their potential customers and preserve them to advance their business.
\end{abstract}

\section{INTRODUCTION}

One of the important things of customer management is how a company be able to preserve their owned customers. In this case, PT. Koba metal Indonesia. as a roll-forming cooperate or the proses of building light-steel into ready-to-use utensils in development section, starts to realize to preserve their customers, considering on the growth of rollforming business who sells same products as them. To prevent the displacement of customers, knowing the potential group of customers is important, so that the company could preserve those potential customers by giving excellent service or prizes. The prizes could be souvenirs that given to their customers annually. PT. Koba metal 
Indonesia. is one of the companies that realize the importance of the connection between loyal customers and the success of company's business.

\section{METHODS}

$\mathrm{K}$-means is an algorithm that be used in partial classification that separate datas into different groups. This algorithm is able to minimalize the gap between data to its cluster. Basically, the application of this algorithm in the clustering process depends on the received datas and the conclusions that expected to be achieved in the end of the process. So in kmeans algorithm application, there are precepts, such as:

a. How many clusters that needed to be inserted.

b. Only have numeric type attribute.

Basically k-means algorithm only takes a piece of those plenty components that is received to become the center of the beginning cluster. After that, the k-means algorithm will examine each of the component in that data population and mark that component into one of defined cluster center depend on the minimum gap between cluster's center to another center. After that, the cluster's center's position will be counted until all of the data components is classified into each of clusters and at last, new cluster will be built.

Data mining is a data analyse to discover an obvious relation and conclude the unknown with the current method that useful and understood by the owner.

Clustering or classifying is a method that is used to divide data circuits into some groups based on their similarity which has determined before. Cluster is a group of similar data in the same, cluster, and dissimilar to other cluster's object. Object will be classified into one or more clusters, so that objects in a cluster have substantial similarity between one and another.

\section{RESULTS}

The image below is an applied k-means algorithm method's layout on rapidminer. This process starts in Read excel step, which is an excel files in clustering data are processed with k-means algorithm, after that, these datas will be on apply model stage, to adjust these k-means algorithm clusterised data to enter performance stage. On performance stage, these datas will be processed to produce PerformaceVector, cluster model, and example set output.

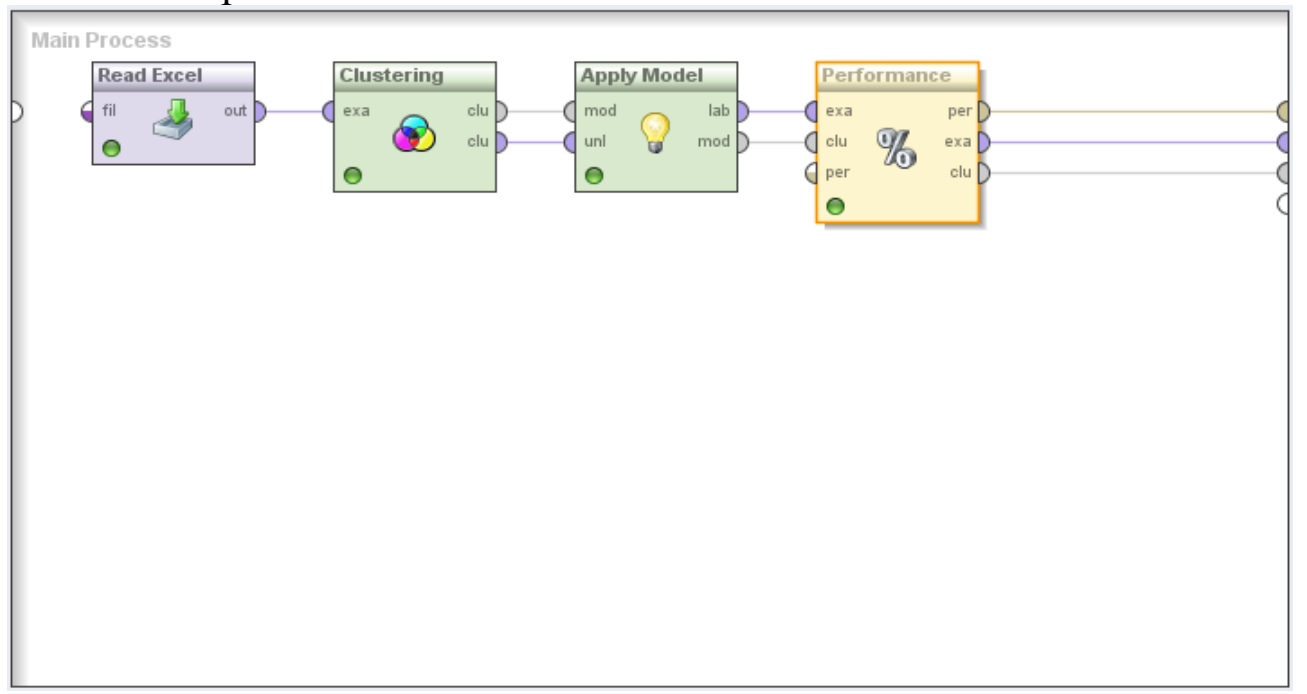

Image 1: Algorithm K-Means Application on Rapidminer 


\section{Cluster Model}

Cluster 0: 9 items Cluster 1: 436 items

Cluster 2: 1 items

Total number of items: 446

Image 2: Cluster Model on Rapidminer

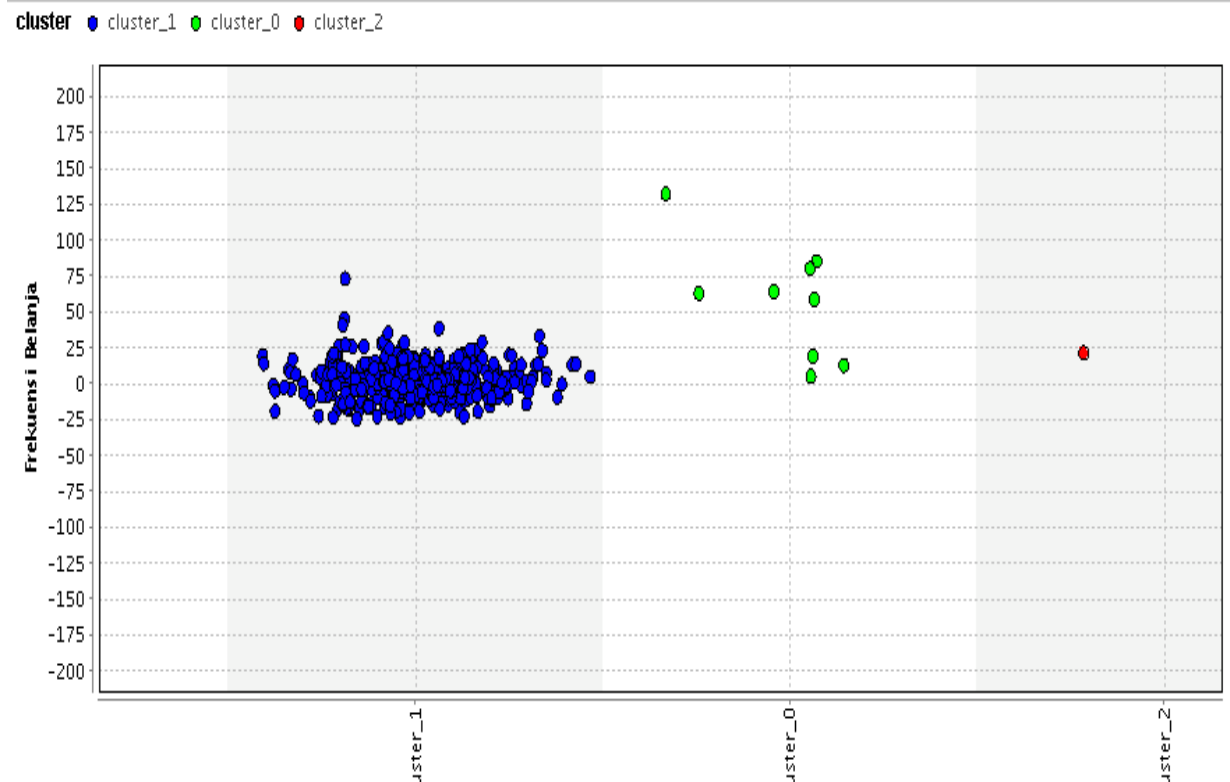

Gambar 3: K-Means Diagram on Rapidminer

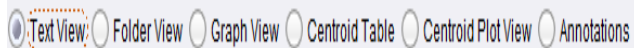

\section{Cluster Yodel}

Cluster 0: 9 items

Cluster 1: 436 items

Cluster 2: 1 items

Total number of items: 446

\section{Gambar 4: Cluster Model on Rapidminer}


Table 1. Comparison Value on 3 Algorithm Methods

\begin{tabular}{|c|c|c|c|}
\hline & Kr-Means & Naive Bayes & Algoritma C45 \\
\hline Accuracy & $93 \%$ & $90.06 \%$ & $84.30 \%$ \\
\hline $\begin{array}{l}\text { Data } \\
\text { Source }\end{array}$ & $\begin{array}{l}\text { PT. Koba } \\
\text { Indonesia } \\
\text { Datal }\end{array}$ & $\begin{array}{ll}\text { PI. Koba } & \text { Metal } \\
\text { Indonesia } & \text { Sales } \\
\text { Data } & \\
\end{array}$ & $\begin{array}{l}\text { PT. Koba Metal } \\
\text { Indonesia Sales } \\
\text { Data }\end{array}$ \\
\hline $\begin{array}{l}\text { Secondary } \\
\text { Algorithm }\end{array}$ & & & \\
\hline
\end{tabular}
compilations, such as low, medium, and high, which is showed on image 2 and 3. Each of compilation's affliation's functions are formulated as follows: Based on table above, can be known that Naïve Bayes accuracy value is $90.06 \%, 84,30 \%$ for $\mathrm{C} 4.5$, and $93 \%$ for K-Means. These 3 methods above examine PT. Kobe metal Indonesia.'s sales data. For K-Means accuracy value is obtained

\section{DISCUSSION}

Decision making is started by cluster determination stage, which is done based on the aim of data mining process. On this case, data that is aimed to be generated is potential customer's data, with 3 categories, such as potential, less potential, and not potential. Can be concluded that the built clusters are $3 / \mathrm{c}=3$. the cluster central point could be decided independently or by find out the hight value (c1), average value(c2), and lowest value (c3). Here is data example with 40 datas taken. by manual calculation with the formula as follows:

$$
\text { Acuracy }=\frac{T P+T N}{T P+T N+F P+F N}
$$

From algorithm k-means process' result's data, could be indicated 9 on potential customer category, 1 for less potential customers, and 436 for not potential customers. Compared with the raw data (have not been processed with k-means algorithm) has been predicted 16 potential customers, 20 less potential customers, and 410 not potential customers. These type of data later gained the values that will be used to count the accuracy value, as the table follows:

Table 2. Accuracy Point

\begin{tabular}{|c|c|}
\hline TP (True Positif) & FP (False Positif) \\
\hline $\mathbf{4 l 5}$ & $\mathbf{3 1}$ \\
\hline FN (False Negatif) & TN (True Negatif) \\
\hline $\mathbf{0}$ & $\mathbf{0}$ \\
\hline
\end{tabular}

Table 3. Sales Data Sample on PT. KMI

\begin{tabular}{|c|c|c|c|}
\hline No & CUSTOMER & $\begin{array}{c}\text { FREKUENSI } \\
\text { BELAN } \\
\text { JA }\end{array}$ & TOTAL BELANJA \\
\hline 1 & 3 SAUDARA,TB & 1 & $4,800,000$ \\
\hline 2 & AAN SUTARYA,BPK & 2 & $2,112,500$ \\
\hline 3 & AAN,BPK & 3 & $1,551,900$ \\
\hline 4 & AANG,BPK & 8 & $50,922,500$ \\
\hline 5 & AAT, BPK & 4 & $7,336,000$ \\
\hline 6 & ABADI JAYA & 6 & $25,945,450$ \\
\hline 7 & ABDUL KODIR GRAHAM & 2 & $74,500,000$ \\
\hline 8 & ACEP TRALIIS CITRA & 1 & 256,250 \\
\hline 9 & ACEP, BPK & 4 & $21,334,000$ \\
\hline 10 & ACIM, BPK & 3 & $21,175,500$ \\
\hline 11 & ACONG,BPK & 2 & $48,226,150$ \\
\hline 12 & ADANG, BPK & 3 & $6,366,930$ \\
\hline 13 & ADE SAPUTRA,BPK & 8 & $53,004,000$ \\
\hline 14 & $\mathrm{ADE}, \mathrm{BPK}$ & 8 & $83,664,000$ \\
\hline 15 & ADI, BPK & 5 & $54,771,940$ \\
\hline 16 & ADIH,BPK & 5 & $12,507,250$ \\
\hline 17 & ADDN,BPK & 4 & $55,035,000$ \\
\hline 18 & ADNO,BPK & 1 & 375,000 \\
\hline 19 & $\mathrm{AEP}, \mathrm{BPK}$ & 1 & $12,185,000$ \\
\hline 20 & AFANG, BPK & 2 & $12,973,000$ \\
\hline 21 & AFIN,BPK & 1 & $1,764,000$ \\
\hline 22 & AGI / ADN, $\mathrm{PT}$ & 1 & $29,475,000$ \\
\hline 23 & AGUS LAS,BPK & 8 & $6,448,475$ \\
\hline 24 & AGUS SUHAEMI,BPK & 1 & $1,570,000$ \\
\hline 25 & AGUS,BPK & 7 & $16,752,200$ \\
\hline 26 & AHMAAD,BPK & 4 & $12,697,500$ \\
\hline 27 & AHIMD, BPK/BU LIA & 1 & $9,552,500$ \\
\hline 28 & AHOK,BPK & 2 & $32,675,600$ \\
\hline 29 & AKUR REHA,CV & 5 & $33,253,600$ \\
\hline 30 & ALAN,BPK & 3 & $15,400,000$ \\
\hline 31 & ALAY,BPK & 1 & $7,872,975$ \\
\hline 32 & ALDI, BPK & 1 & 888,000 \\
\hline 33 & ALEX,BPK & 1 & $5,923,800$ \\
\hline 34 & ALFA MIDI & 1 & $4,630,500$ \\
\hline 35 & ALFAUZAN, TB & 2 & $10,380,000$ \\
\hline 36 & ALI, BPK / IRON GALERY TK. & 2 & $10,374,900$ \\
\hline 37 & ALNO,TB & 1 & $14,500,600$ \\
\hline 38 & ALUNG,BPK & 3 & $27,975,500$ \\
\hline 39 & AMAN,BPK & 5 & $5,479,000$ \\
\hline 40 & AMANAH PRIMA INDONESIA.PT & 3 & $6.490,040$ \\
\hline
\end{tabular}

Cluster center point:

$\mathrm{C} 1=$ highest value (shopping frequency, total expenditure)

$\mathrm{C} 1=(8,83664000)$ 
$\mathrm{C} 2=$ average value (shopping frequency, total expenditure)

$\mathrm{C} 2=(3.15,20078664)$

C3=lowest value (shopping frequency, total expenditure)

$\mathrm{C} 3=(1,256250)$

Calculate the gap between data to cluster central point.

Table 4. Iteration 1 Process Result

\begin{tabular}{|c|c|c|c|c|c|c|}
\hline No & CUSTOMER & $\begin{array}{c}\text { FREKUENSI } \\
\text { BELANJA }\end{array}$ & $\begin{array}{c}\text { TOTAL } \\
\text { BELANJA }\end{array}$ & Cl & $\mathrm{C}_{2}$ & C3 \\
\hline 1 & 3 SAUDARA,TB & 1 & $4,800,000$ & & & * \\
\hline 2 & AAN SUTARYA,BPK & 2 & $2,112,500$ & & & $*$ \\
\hline 3 & AANBPK & 3 & $1,551,900$ & & & $*$ \\
\hline 4 & AANG,BPK & 8 & $50,922,500$ & & $*$ & \\
\hline 5 & AAT, BPK & 4 & $7,336,000$ & & & $*$ \\
\hline 6 & ABADI JAYA & 6 & $25,945,450$ & & $*$ & \\
\hline 7 & ABDUL KODIR GRAHAM & 2 & $74,500,000$ & * & & \\
\hline 8 & ACEP TRALIS CITRA & 1 & 256,250 & & & $*$ \\
\hline 9 & ACEP, BPK & 4 & $21,334,000$ & & $*$ & \\
\hline 10 & ACIM, BPK & 3 & $21,175,500$ & & $*$ & \\
\hline 11 & ACONG,BPK & 2 & $48,226,150$ & & $=$ & \\
\hline 12 & ADANG, BPK & 3 & $6,366,930$ & & & $*$ \\
\hline 13 & ADE SAPUTRA,BPK & 8 & $53,004,000$ & 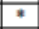 & & \\
\hline 14 & $A D E, B P K$ & 8 & $83,664,000$ & $=$ & & \\
\hline 15 & ADI, BPK & 5 & $54,771,940$ & \begin{tabular}{|l|l|}
$*$ \\
\end{tabular} & & \\
\hline 16 & ADIH,BPK & 5 & $12,507,250$ & & $*$ & \\
\hline 17 & ADIN,BPK & 4 & $55,035,000$ & $*$ & & \\
\hline 18 & ADNO,BPK & 1 & 375,000 & & & $*$ \\
\hline 19 & AEP,BPK & 1 & $12,185,000$ & & $*$ & \\
\hline 20 & AFANG, BPK & 2 & $12,973,000$ & & $*$ & \\
\hline 21 & AFIN,BPK & 1 & $1,764,000$ & & & $*$ \\
\hline 22 & AGI / ADIN,PT & 1 & $29,475,000$ & & $*$ & \\
\hline 23 & AGUS LAS,BPK & 8 & $6,448,475$ & & & * \\
\hline 24 & AGUS SUHAEMI,BPK & 1 & $1,570,000$ & & & * \\
\hline 25 & AGUS,BPK & 7 & $16,752,200$ & & $*$ & \\
\hline 26 & AHMAD,BPK & 4 & $12,697,500$ & & 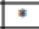 & \\
\hline 27 & AHMID, BPK/BU LIA & 1 & $9,552,500$ & & & * \\
\hline 28 & AHOK,BPK & 2 & $32,675,600$ & & $*$ & \\
\hline 29 & AKUR REHA,CV & 5 & $33,253,600$ & & $=$ & \\
\hline 30 & ALAN,BPK & 3 & $15,400,000$ & & $*$ & \\
\hline 31 & ALAY,BPK & 1 & $7,872,975$ & & & * \\
\hline 32 & ALDI, BPK & 1 & 888,000 & & & $*$ \\
\hline 33 & ALEX,BPK & 1 & $3,923,800$ & & & $*$ \\
\hline 34 & ALFA MIDI & 1 & $4,630,500$ & & & $*$ \\
\hline 35 & ALFAUZAN,TB & 2 & $10,380,000$ & & $*$ & \\
\hline 36 & ALI, BPK / IRON GALERY TK. & 2 & $10,374,900$ & & $*$ & \\
\hline 37 & ALNO,TB & 1 & $14,500,600$ & & 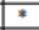 & \\
\hline 38 & ALUNG,BPK & 3 & $27,975,500$ & & $=$ & \\
\hline 39 & AMAN,BPK & 5 & $5,479,000$ & & & * \\
\hline 40 & AMANAH PRIMA INDONESIA,PT & 3 & $6,490,040$ & & & * \\
\hline
\end{tabular}

Calculate the cluster center again with the current cluster's membership. The new cluster center is the average of all datas/objects in certain group.

Finding the new $\mathrm{C} 1$

$$
\begin{aligned}
& C_{11}=\frac{(2+8+8+5+4)}{5}=5.4 \\
& C_{12}=\frac{(74500000+53004000+83664000+54771940+55035000)}{4}=64194988
\end{aligned}
$$

So the new C1 center is $=5.4,64194988$

$$
\begin{aligned}
& C_{21}=\frac{61}{18}=3.4 \\
& C_{22}=\frac{408753750}{18}=22708541.67
\end{aligned}
$$

So the new C2 center is $=3.4,22708541.67$

$$
\begin{aligned}
& C_{31}=\frac{38}{17}=2.2 \\
& C_{32}=\frac{73417870}{17}=4318698.24
\end{aligned}
$$

So the new C3 center is $=2.2,4318698.24$

After getting the new cluster result, count the gap between data and the new cluster center's

\begin{tabular}{|c|c|c|c|c|c|c|}
\hline No & CUSTOMER & $\begin{array}{l}\text { FREKUENSI } \\
\text { BELANJA }\end{array}$ & $\begin{array}{c}\text { TOTAL } \\
\text { BELANJA }\end{array}$ & $\mathrm{Cl}$ & C2 & C3 \\
\hline 1 & 3 SAUDARA, TB & 1 & $4,800,000$ & & & $*$ \\
\hline 2 & AAN SUTARYA,BPK & 2 & $2,112,500$ & & & \begin{tabular}{|l}
$*$ \\
\end{tabular} \\
\hline 3 & AAN,BPK & 3 & $1,551,900$ & & & $*$ \\
\hline 4 & AANG,BPK & 8 & $50,922,500$ & $*$ & & \\
\hline 5 & AAT, BPK & 4 & $7,336,000$ & & & ${ }^{*}$ \\
\hline 6 & ABADI JAYA & 6 & $25,945,450$ & & * & \\
\hline 7 & ABDUL KODIR GRAHAM & 2 & $74,500,000$ & * & & \\
\hline 8 & ACEP TRALIS CITRA & 1 & 256,250 & & & $*$ \\
\hline 9 & ACEP, BPK & 4 & $21,334,000$ & & $*$ & \\
\hline 10 & ACIM, BPK & 3 & $21,175,500$ & & * & \\
\hline 11 & $A C O N G, B P K$ & 2 & $48,226,150$ & * & & \\
\hline 12 & ADANG, BPK & 3 & $6,366,930$ & & & $*$ \\
\hline 13 & ADE SAPUTRA,BPK & 8 & $53,004,000$ & $*$ & & \\
\hline 14 & $A D E, B P K$ & 8 & $83,664,000$ & * & & \\
\hline 15 & ADI, BPK & 5 & $54,771,940$ & * & & \\
\hline 16 & ADIH,BPK & 5 & $12,507,250$ & & & $*$ \\
\hline 17 & $A D I N, B P K$ & 4 & $55,035,000$ & $*$ & & \\
\hline 18 & ADINO,BPK & 1 & 375,000 & & & \begin{tabular}{|c|}
$*$ \\
\end{tabular} \\
\hline 19 & AEP,BPK & 1 & $12,185,000$ & & & $*$ \\
\hline 20 & AFANG, BPK & 2 & $12,973,000$ & & & $*$ \\
\hline 21 & AFIN,BPK & 1 & $1,764,000$ & & & $*$ \\
\hline 22 & AGI / ADIN,PT & 1 & $29,475,000$ & & $*$ & \\
\hline 23 & AGUS LAS,BPK & 8 & $6,448,475$ & & & \begin{tabular}{|l|l}
$*$ & \\
\end{tabular} \\
\hline 24 & AGUS SUHAEMI,BPK & 1 & $1,570,000$ & & & $*$ \\
\hline 25 & AGUS,BPK & 7 & $16,752,200$ & & $*$ & \\
\hline 26 & AHMAD,BPK & 4 & $12,697,500$ & & & \begin{tabular}{|c|}
$*$ \\
\end{tabular} \\
\hline 27 & AHMID, BPK/BU LIA & 1 & $9,552,500$ & & & $*$ \\
\hline 28 & AHOK,BPK & 2 & $32,675,600$ & & * & \\
\hline 29 & AKUR REHA,CV & 5 & $33,253,600$ & & * & \\
\hline 30 & ALAN,BPK & 3 & $15,400,000$ & & $*$ & \\
\hline 31 & AL.AY,BPK & 1 & $7,872,975$ & & & $*$ \\
\hline 32 & ALDI, BPK & 1 & 888,000 & & & $*$ \\
\hline 33 & ALEX,BPK & 1 & $5,923,800$ & & & $*$ \\
\hline 34 & ALFA MIDI & 1 & $4,630,500$ & & & $*$ \\
\hline 35 & ALFAUZAN,TB & 2 & $10,380,000$ & & & $*$ \\
\hline 36 & ALI, BPK / IRON GALERY TK. & 2 & $10,374,900$ & & & * \\
\hline 37 & ALNO,TB & 1 & $14,500,600$ & & $*$ & \\
\hline 38 & ALUNG,BPK & 3 & $27,975,500$ & & $*$ & \\
\hline 39 & AMAN,BPK & 5 & $5,479,000$ & & & \begin{tabular}{|c|}
$*$ \\
\end{tabular} \\
\hline 40 & AMANAH PRIMA INDONESLA,PT & 3 & $6,490,040$ & & & $*$ \\
\hline
\end{tabular}
point, and categorize again the cluster. Repeat those stage all over again until cluster's position on the cluster grouping stage will not change anymore.

Table 5. Iteration 2 Process Result

Table 6. Iteration 3 Process Result 


\begin{tabular}{|c|c|c|c|c|c|c|}
\hline No & CUSTOMER & $\begin{array}{c}\text { FREKUENSI } \\
\text { BELANJA }\end{array}$ & $\begin{array}{c}\text { TOTAL } \\
\text { BELANJA }\end{array}$ & $\mathrm{Cl}$ & C2 2 & C3 \\
\hline 1 & 3 SAUDARA,TB & 1 & $4,800,000$ & & & * \\
\hline 2 & AAAN SUTARYA,BPK & 2 & $2,112,500$ & & & $*$ \\
\hline 3 & AAN,BPK & 3 & $1,551,900$ & & & * \\
\hline 4 & A.ANG,BPK & 8 & $50,922,500$ & * & & \\
\hline 5 & AAT, BPK & 4 & $7,336,000$ & & & * \\
\hline 6 & ABADI JAYA & 6 & $25,945,450$ & & * & \\
\hline 7 & ABDUL KODIR GRAHAM & 2 & $74,500,000$ & * & & \\
\hline 8 & ACEP TRALIS CITRA & 1 & 256,250 & & & * \\
\hline 9 & ACEP, BPK & 4 & $21,334,000$ & & * & \\
\hline 10 & ACIM, BPK & 3 & $21,175,500$ & & $*$ & \\
\hline 11 & ACONG,BPK & 2 & $48,226,150$ & * & & \\
\hline 12 & ADANG, BPK & 3 & $6,366,930$ & & & ${ }^{*}$ \\
\hline 13 & ADE SAPUTRA,BPK & 8 & $53,004,000$ & $*^{*}$ & & \\
\hline 14 & ADE,BPK & 8 & $83,664,000$ & * & & \\
\hline 15 & ADI, BPK & 5 & $54,771,940$ & ${ }^{*}$ & & \\
\hline 16 & ADIH,BPK & 5 & $12,507,250$ & & & ${ }^{*}$ \\
\hline 17 & ADIN,BPK & 4 & $55,035,000$ & * & & \\
\hline 18 & ADINO,BPK & 1 & 375,000 & & & * \\
\hline 19 & AEP,BPK & 1 & $12,185,000$ & & & * \\
\hline 20 & AFANG, BPK & 2 & $12,973,000$ & & & * \\
\hline 21 & AFIN,BPK & 1 & $1,764,000$ & & & * \\
\hline 22 & AGI/ADIN,PT & 1 & $29,475,000$ & & $*$ & \\
\hline 23 & AGUS LAS,BPK & 8 & $6,448,475$ & & & * \\
\hline 24 & AGUS SUHAEMI,BPK & 1 & $1,570,000$ & & & * \\
\hline 25 & AGUS,BPK & 7 & $16,752,200$ & & * & \\
\hline 26 & AHMAD,BPK & 4 & $12,697,500$ & & & * \\
\hline 27 & AHMID, BPK/BUU LIA & 1 & $9,552,500$ & & & * \\
\hline 28 & AHOK,BPK & 2 & $32,675,600$ & & * & \\
\hline 29 & AKUR REHA,CV & 5 & $33,253,600$ & & * & \\
\hline 30 & AL.AN,BPK & 3 & $15,400,000$ & & & * \\
\hline 31 & ALAY,BPK & 1 & $7,872,975$ & & & $*$ \\
\hline 32 & ALDI, BPK & 1 & 888,000 & & & ${ }^{*}$ \\
\hline 33 & ALEX,BPK & 1 & $5,923,800$ & & & * \\
\hline 34 & ALFA MIII & 1 & $4,630,500$ & & & $*$ \\
\hline 35 & ALFAUZAN,TB & 2 & $10,380,000$ & & & ${ }^{*}$ \\
\hline 36 & ALI, BPK / IRON GALERY TK. & 2 & $10,374,900$ & & & * \\
\hline 37 & ALNO,TB & 1 & $14,500,600$ & & & ${ }^{*}$ \\
\hline 38 & ALUNG,BPK & 3 & $27,975,500$ & & * & \\
\hline 39 & AMAN,BPK & 5 & $5,479,000$ & & & ${ }^{*}$ \\
\hline 40 & AMANAH PRIMA INDONESIA,PT & 3 & $6,490,040$ & & & * \\
\hline
\end{tabular}

Table 7. Iteration 4 Process Result

\begin{tabular}{|c|c|c|c|c|c|c|}
\hline No & CUSTOMER & \begin{tabular}{|c|} 
FREKUENSI \\
BELANJA
\end{tabular} & $\begin{array}{c}\text { TOTAL } \\
\text { BELANJA }\end{array}$ & $\mathrm{Cl}$ & $\mathrm{C} 2$ & C3 \\
\hline 1 & 3 SAUDARA,TB & 1 & $4,800,000$ & & & * \\
\hline 2 & A.AN SUTARYA,BPK & 2 & $2,112,500$ & & & * \\
\hline 3 & A.AN,BPK & 3 & $1,551,900$ & & & * \\
\hline 4 & AANG,BPK & 8 & $50,922,500$ & ${ }^{*}$ & & \\
\hline 5 & AAT, BPK & 4 & $7,336,000$ & & & * \\
\hline 6 & ABADI JAYA & 6 & $25,945,450$ & & $*^{*}$ & \\
\hline 7 & ABDUL KODIR GRAHAM & 2 & $74,500,000$ & ${ }^{*}$ & & \\
\hline 8 & ACEP TRALIS CITRA & 1 & 256,250 & & & ${ }^{*}$ \\
\hline 9 & ACEP, BPK & 4 & $21,334,000$ & & $*^{*}$ & \\
\hline 10 & ACIM, BPK & 3 & $21,175,500$ & & * & \\
\hline 11 & ACONG,BPK & 2 & $48,226,150$ & $*$ & & \\
\hline 12 & ADANG, BPK & 3 & $6,366,930$ & & & * \\
\hline 13 & ADE SAPUTRA,BPK & 8 & $53,004,000$ & ${ }^{*}$ & & \\
\hline 14 & $\mathrm{ADE}, \mathrm{BPK}$ & 8 & $83,664,000$ & $*$ & & \\
\hline 15 & ADI, BPK & 5 & $54,771,940$ & ${ }^{*}$ & & \\
\hline 16 & ADIH,BPK & 5 & $12,507,250$ & & & * \\
\hline 17 & $A D I N, B P K$ & 4 & $55,035,000$ & ${ }^{*}$ & & \\
\hline 18 & ADINO,BPK & 1 & 375,000 & & & $*^{*}$ \\
\hline 19 & AEP,BPK & 1 & $12,185,000$ & & & * \\
\hline 20 & AFANG, BPK & 2 & $12,973,000$ & & & ${ }^{*}$ \\
\hline 21 & AFIN,BPK & 1 & $1,764,000$ & & & * \\
\hline 22 & AGI / ADIN,PT & 1 & $29,475,000$ & & ${ }^{*}$ & \\
\hline 23 & AGUS LAS,BPK & 8 & $6,448,475$ & & & ${ }^{*}$ \\
\hline 24 & AGUS SUHAEMIBPK & 1 & $1,570,000$ & & & * \\
\hline 25 & AGUS,BPK & 7 & $16,752,200$ & & ${ }^{*}$ & \\
\hline 26 & AHMAD,BPK & 4 & $12,697,500$ & & & * \\
\hline 27 & AHMID, BPK/IBU LIA & 1 & $9,552,500$ & & & * \\
\hline 28 & AHOK,BPK & 2 & $32,675,600$ & & $*$ & \\
\hline 29 & AKUR REHA,CV & 5 & $33,253,600$ & & $*$ & \\
\hline 30 & ALAN,BPK & 3 & $15,400,000$ & & & * \\
\hline 31 & ALAY,BPK & 1 & $7,872,975$ & & & * \\
\hline 32 & ALDI, BPK & 1 & 888,000 & & & * \\
\hline 33 & ALEX,BPK & 1 & $5,923,800$ & & & * \\
\hline 34 & ALFA MIDI & 1 & $4,630,500$ & & & * \\
\hline 35 & \begin{tabular}{|l} 
ALFAUZAN,TB \\
\end{tabular} & 2 & $10,380,000$ & & & * \\
\hline 36 & ALI, BPK / IRON GALERY TK. & 2 & $10,374,900$ & & & ${ }^{*}$ \\
\hline 37 & ALNO,TB & 1 & $14,500,600$ & & & * \\
\hline 38 & ALUNG,BPK & 3 & $27,975,500$ & & $*^{*}$ & \\
\hline 39 & AMAN,BPK & 5 & $5,479,000$ & & & * \\
\hline 40 & AMANAH PRIMA INDONESIA,PT & 3 & $6,490,040$ & & & $*$ \\
\hline
\end{tabular}

Because on the $3^{\text {rd }}$ and the $4^{\text {th }}$ iteration has no change of the cluster postion, then the process is stopped. It is known that on the $4^{\text {th }}$ iteration, cluster 1 central point is on $(5.3,60017656)$. So that could be cluster central point concluded that cluster 1's customers are potential customers, for cluster 2's central point is on $(3.9,26073356)$ categorized as less potential customers, and for the cluster 3's central point is on $(2.3,6977445)$ categorized as not potential customers.

\section{CONCLUSION}

Based on the research that has been done, it can be concluded as follows:

1. Agglomeration of the kmi co.'s sales data could be done by clustering model, with agglomerating those datas into categories basen on data's similarity in a category.

2. To get potential customers data by kmeans algorithm is done by these stages as follows:

a. Decide the cluster's amount.

b. Decide the cluster's center point.

c. Calculate the gap between data to cluster's center point.

d. Agglomerate data into clusters depend on the shortest gap or the smallest.

e. Repeat step b-d, and compare the data position on every result. If the data position changes, repeat step $b-d$ all over again. If the position co not change anymoe, the process is done.

f. The potential customer determination is seen by the biggest cluster's center poin from the built on the last stage when the data position on the cluster stays still.

3. Getting potential customer data with Rapidminer application is done by importing the first data to be processed can be in the form of an excel or csv module read excel if excel file and read csv if shaped csv file. The data processed includes the name of the customer (customer), shopping frequency, and total expenditure. after that input K-Means algortima module and also change the parameters such as the number of clusters desired. Then connect the module read 
excel / csv to K-means algorithm module and forwarded to the endpoint that is already available. And if it is done, the next data can be processed by Rapidminer application that produces clusters with data that has been grouped and also displays the highest cluster central point value so that it can be determined which cluster into the category of potential customers. 


\section{REFERENCES}

Aji Supriyanto, "Pengantar teknologi Informasi”, Jakarta: Salemba Infotek,2005.

Amstrong, Gary \& Philip, Kotler. 2002. Dasar-dasar Pemasaran. Jilid 1, Alih Bahasa Alexander Sindoro dan Benyamin Molan. Jakarta: Penerbit Prenhalindo.

Andayani, Sri. (2007). Pembentukan cluster dalam Knowledge Discovery in Database dengan Algoritma K-Means. Jurnal, semnasIF 2007. Yogyakarta: Fakultas Matematika dan Pendidikan Matematika Universitas Negeri Yogyakarta.

Binanto, Iwan (2010). Multimedia Digital - Dasar Teori dan Pengembangannya. Yogyakarta.

Blattberg, Robert C., Getz, Gary, Thomas, Jacquelyn S. (2001). Customer Equity: Building and Managing Relationships as Valuable Assets. Harvard Business Scholl Publishing.

Connolly, Thomas and Begg, Carolyn, 2010, Database Systems: A Practical Approach to Design, Implementation, and Management, Fifth Edition. Pearson Education, Boston.

Davis Kadir. (2003). Informasi Komputer. Andi Offset, Yogyakarta.

Gregory M. Scott, 1986, Prcinciples of Management Information Systems, McGraw-Hill, New York.

Han, Jiawei dan Kamber, Micheline. (2006), Data Mining : Concept and Techniques Second Edition, Morgan Kaufmann Publishers.

Harip Santoso. 2004. Membuat Aplikasi Menggunakan Visual Basic 6.0. PT Elex Media Komputindo. Jakarta.

Hoffer, Jeffrey A., Prescott Mary B., Mcfadden, Fred R. 2007. Modern Database Management. New Jersey : Pearson Prentice Hall.

Indonesian Registrant Domain and WebHosting. (2013, 22 Maret). Info Pengertian Dari PHP. Diperoleh pada 26 Maret 2014, dari http://www.idreg.net/pengertian-dari-php.

John Burch dan Gary Grudnitski, 1986, Information Systems Theory and Practice, John Wiley and Sons, New York.

Kristanto, A. (2008). Perancangan Sistem Informasi dan Aplikasinya. Yogyakarta: Gava Media.

Kurgan, L., and Musilek, P. 2006. A survey of knowledge discovery and data mining process models. Knowledge Engineering Review, 21(1):1-24.

Mariscal, Marba'n dan Ferna'ndes.2010. A Survey Of Data Mining And Knowledge Discovery Models And Methodologies. The Knowledge Engineering Review, 25(2), 137-166. http://dx.doi.org.

Pressman, Roger S. (2005). Software Engineering A Practitioner's Approach. Sixth Edition. New York: McGraw-Hill.

Plaisant, S. B. (2005). Designing the user interface 4th Edition : strategies for effective humancomputer interaction. England: Pearson Addison Wesley Publishing Company Inc.

Robert J. Verzello dan John Reuter III, (1982), "Data Processing: Systems and Concepts”, Tokyo: Mc Graw-Hill Kogakusha, Ltd.

Santoso, Insap (2010). Interaksi Manusia dan Komputer Edisi 2. Yogyakarta: Andi Publisher

S. Agustina, D. Yhudo, H. Santoso, N. Marnasusanto, A. Tirtana dan F. Khusnu, "Clustering Kualitas Beras Berdasarkan Ciri Fisik Menggunakan Metode K-Means," Universitas Brawijaya Malang, Malang, 2012.

Universitas Brawijaya. (2013, 26 Februari). Pengertian dari OOP, Class, Method, Behavior dan Constructor. Diperoleh pada 26 Maret 2014, dari http://blog.ub.ac.id/home/pengertian-darioop-class-method-behavior-dan-constructor/comment-page-1.

Widodo, Prabowo.P,Dkk, 2011, Pemodelan Sistem Berorientasi Obyek A. Dengan UML, Graha ilmu, Yogyakarta.

Yulikuspartono. 2009. Pengantar Logika dan Algoritma. Yogyakarta: Andi Offset. 


\section{BIOGRAPHY}

Sandi Kristianto - Bachelor of Computer Science (S1) Informatic Engineering (Database), 2017. working at PT. Koba Metal Indonesia.

Yusuf Kurnia - Bachelor of Computer Science (S1) Information System (Corporate System), 2009, Master of Computer Science (S2) Information System Technology, 2013. Currently active as a Permanent Lecturer in Informatics Engineering Program, Buddhi Dharma University. 